\title{
Experimental study on photoprotective activity of cerium dioxide nanoparticles cream on photosensibilized skin
}

\author{
Ganna V. Zaychenko ${ }^{1}$, Vikola O. Lyapunov², Vladimir S. Yefanov ${ }^{3}$, Oksana A. Pokotylo ${ }^{1}$, Pavlo \\ V. Simonov ${ }^{1}$ \\ ${ }^{I}$ Department of Pharmacology, Bogomolets National Medical University, Ukraine, ${ }^{2}$ Scientific Institution "Institute for \\ Single Crystals" of Academy of NASU, Ukraine, ${ }^{3} L L C$ "Nanomedtech", Ukraine
}

Background. Ultraviolet (UV) irradiation, which is a well known carcinogen, is among the main risk factors of basal cell carcinoma, squamous cell carcinoma, and melanoma development. It poses the greatest threat for photosensibilized skin. Photosensitization is caused by medicinal substances, some food products, and household chemicals. It is necessary to develop drugs for topical use with the photoprotective activity, that may contain cerium dioxide nanoparticles (CeONP) as an active substance. In the study we determined the photoprotective activity of CeONP cream on the model of photodynamic injury on fotosensibilized skin in guinea pigs.

Methods. CeONP cream was developed in Nanomedtech (Kyiv, Ukraine) and Institute for Single Crystals (Kharkiv, Ukraine). The photoprotective activity of the cream was studied on the model of acute photodynamic skin inflammation in guinea pigs. UV erythema was obtained with the use of UV emitter of type OKH-011M in the wavelength range of 240 $-320 \mathrm{~nm} .0 .3 \%$ ammifurin solution was applied as a photosensitizer in the amount of $1 \mathrm{ml} / 1$ depilated area of the skin 1 hour before irradiation. The photoprotective activity was evaluated by dermatoscopy results and following indices: skin temperature dynamics, leukocyte count and histamine level in blood, prooxidative-antioxidative skin status, and prostaglandin F2 $\alpha$ level in skin.

Results. During the study an erythema of different severity developed in animals. The changes in following indices, which confirmed the photoprotective activity of CeONP cream, were observed: decrease in photodermatitis' signs, normalization of leukocyte count and histamine levels in blood, decrease in local skin hyperthermia, and increase in reduced glutathione levels and catalase activity with simultaneous decrease in diene conjugate, thiobarbituric acid reactant, and prostaglandin F2 $\alpha$ levels in skin homogenate.

Conclusions. The obtained results proved the presence of anti-inflammatory, antioxidant types of action of CeONP cream, which were at the basis of its photoprotective activity. It is expedient to continue non-clinical studies of this dosage form with the aim of its introduction into medical practice as the photoprotective drug. 